\title{
Abundances of Potassium, Argon, and Sulphur in Solar Flares
}

\author{
K. J. H. Phillips \\ National Research Council Senior Research Associate, NASA Goddard \\ Space Flight Center, Code 682, Greenbelt, MD 20771, USA
}

J. Sylwester and B. Sylwester

Space Research Centre, Polish Academy of Sciences, ul. Kopernika 11, 51-622 Wroctaw, Poland

\section{E. Landi}

Artep Inc., Ellicott City, MD 21042, USA and E. O. Hulburt Center for Space Research, US Naval Research Laboratory, Washington DC 20375, $U S A$

\begin{abstract}
Observations of the 3.3-6.1 $\AA$ X-ray line and continuous spectrum during four long-duration flares with the RESIK crystal spectrometer on the Coronas- $F$ spacecraft have been analyzed to get the absolute abundances of potassium, argon, and sulphur. A differential emission measure of the form $D E M \propto \exp \left(-T_{e} / T_{0}\right)$ was found to give the most consistent results of three models including an isothermal model. We obtained $\mathrm{K} / \mathrm{H}=(3.7 \pm 1.0) \times 10^{-7}$, a factor 3 times photospheric; $\mathrm{Ar} / \mathrm{H}=(2.8 \pm 0.2) \times 10^{-6}$, slightly lower than photospheric; and $\mathrm{S} / \mathrm{H}$ $=(2.2 \pm 0.4) \times 10^{-5}$, approximately equal to photospheric. These measurements are consistent with a pattern in which elements with low $(<10 \mathrm{eV})$ first ionization potential are enriched in the corona by a factor of about 3 and elements of high first ionization potential have abundances approximately equal to photospheric.
\end{abstract}

\section{Introduction}

Measurements of solar gamma-ray line emission, energetic particles, and X-ray and ultraviolet line emission have indicated that the coronal abundances of elements having low first ionization potential (FIP), i.e. $<10 \mathrm{eV}$, are higher by a factor 3 or 4 than photospheric, but those of elements having high FIP have abundances either approximately photospheric or slightly lower (see the review by Feldman \& Laming 2000). Consequently, simultaneous measurements of line emission with a wide variety of FIPs offer a good means of verifying this so-called FIP effect. To this end, the REntgenowsky Spektrometr s Izognutymi Kristalami (RESIK) X-ray crystal spectrometer, covering lines of highly ionized potassium, argon, and sulphur, was built and launched on the Russian Coronas- $F$ spacecraft. The instrument consists of a pair of spectrometers, A and B, having a 
double position-sensitive proportional counter detecting solar X-rays which are diffracted by two crystals. The crystals are slightly bent convex so that the Bragg diffraction condition $n \lambda=2 d \sin \theta(\theta=$ Bragg angle, $n$ the diffraction order, $\lambda$ wavelength, and $d$ the crystal lattice spacing) varies over the crystal face. RESIK has no collimator, so flares are recorded wherever they occur on the Sun. The optical axis of the instrument is within 5 arcmins of Sun centre normally, and the dispersion direction slowly varies depending on the spacecraft orientation.

Spectrometer A covers the ranges 3.37-3.88 $\AA$ and 3.82-4.33 $\AA$ (channels 1 and 2), and spectrometer $\mathrm{B}$ the ranges 4.31-4.89 $\AA$ and 4.96-6.09 $\AA$ (channels 3 and 4). Channel 1 includes the He-like K (K XVIII) lines $1 s^{2}-1 s n l(n l=2 s, 2 p)$ at 3.532-3.571 $\AA$, and the Ly $\alpha$ lines of H-like Ar (Ar XVIII) at 3.731, $3.737 \AA$; channel 2 includes the $1 s^{2}-1 s n l$ lines of He-like Ar (Ar XVII) at 3.949-3.994 and $1 s^{2}-1 s 3 p, 1 s^{2}-1 s 4 p$ lines of He-like S (S XV) at 4.299, $4.088 \AA$ respectively; channel 3 includes the Ly $\alpha$ lines of H-like S (S XVI) at 4.727, $4.733 \AA$; channel 4 includes several lines including H-like Si (Si XIV) Ly $\beta$ at $5.217 \AA$ and the $1 s^{2}-1 s 3 p, 1 s^{2}-1 s 4 p$ lines of He-like Si (Si XIII) at 5.678, $5.402 \AA$ respectively. Channels 1 and 2 record solar continuum as the instrumental background is very small, but there is a substantial (but in principle known) instrumental background in channels 3 and 4 .

Large flares were recorded by RESIK from the time of the launch of Coronas$F$ on 2001 July 31, and several have detectable K XVIII and Ar XVIII line emission above the flare continuum. This paper reports on the analysis of four long-duration flares, during which line and continuum emission were repeatedly measured and the relative and absolute (i.e. relative to hydrogen) abundances of $\mathrm{K}, \mathrm{Ar}$, and $\mathrm{S}$ determined.

\section{Method of Analysis}

Contribution or $G\left(T_{e}\right)$ functions were determined for all observed lines with the CHIANTI atomic code and database (Dere et al. 1997) using ionization fractions of Mazzotta et al. (1998) and the coronal abundances of Feldman \& Laming (2000). We then made three assumptions for the flare temperature structure at each of the observation times: (A) an isothermal plasma, defined by temperature $T_{e}$; then simple analytic forms for the differential emission measure $D E M,(\mathrm{~B})$ $D E M=\alpha T_{e}^{\beta}$ and (C) $D E M=\alpha \exp \left(-T_{e} / T_{0}\right)$, where $\alpha, \beta$, and $T_{0}$ are free parameters. The measured line ratios defined by the flux of the two Si XIII lines $(5.402,5.678 \AA)$ summed to the $\operatorname{Si}$ XIV $(5.217 \AA)$, were compared with curves of $R_{\mathrm{Si}}$ which is given by $G_{1}\left(T_{e}\right) / G_{2}\left(T_{e}\right)$ ( $G_{1}=$ the summed $G\left(T_{e}\right)$ function of the two Si XIII lines, $G_{2}=$ the $G\left(T_{e}\right)$ function of the Si XIV line) for model (A), but equal to

$$
R_{\mathrm{Si}}=\frac{\int G_{1}\left(T_{e}\right) D E M\left(T_{e}\right) d T_{e}}{\int G_{2}\left(T_{e}\right) D E M\left(T_{e}\right) d T_{e}}
$$

for models (B) and (C). This enabled determinations of $T_{e}$ (model (A)), $\beta$ (model (B)), and $T_{0}$ (model (C)). The parameter $\alpha$ we left undetermined because of the current uncertainty in the absolute sensitivity of RESIK. 
For model (A), we calculated expected ratios $R_{\exp }=G_{3}\left(T_{e}\right) / G_{4}\left(T_{e}\right)$, where $T_{e}$ is the temperature obtained from $R_{\mathrm{Si}}$, and $G_{3}$ and $G_{4}$ are contribution functions of (i) the Ar XVIII lines and K XVIII (resonance or $w$ ) line of channel 1; (ii) the Ar XVII $(w)$ line and two S XV lines of channel 2. For models (B) and (C), $R_{\text {exp }}$ is given by an equation similar to Eq. (1) but with $G_{3}$ and $G_{4}$ substituted for $G_{1}$ and $G_{2}$. If models (A)-(C) accurately describe the flare temperature structure, any differences between the observed line flux ratios $R_{\text {obs }}$ and $R_{\text {exp }}$ will reflect differences in abundance ratios from the coronal values we assumed.

We repeated this analysis for line-to-continuum ratios, using observed ratios in channels 1 and 2 and CHIANTI for the line contribution functions and the equivalent functions describing the continuum.

\section{The Observations}

The four flares observed by RESIK occurred on 2002 April 14/15, July 11, July $22 / 23$, and July $26 / 27$, each lasting several hours, and having GOES importance $\mathrm{M}$ or higher. RESIK spectra are available for over the entire flare decay in time blocks of 4-24 minutes long. Strong Ar XVII, S XV, Si XIII and Si XIV line emission was detected throughout. The K XVIII lines were detectable when the GOES level was above $\mathrm{C} 4$, and the weaker Ar XVIII lines were detectable at $G O E S$ level M1 or higher. The slow decay of each flare enabled us to get repeated measurements of line and line-to-continuum ratios, allowing us to investigate any abundance changes.

Accurate values of the instrument sensitivity are still in progress, so that it is not yet possible to derive precise values of line and continuum fluxes, but the relative sensitivity over each channel is known and so we are able to obtain line flux ratios K XVIII/Ar XVII, Ar XVII/S XV, and Si XIII/Si XIV. For K XVIII and Ar XVII, we took the resonance line $w$ only. From the Si ratios we were able to get electron temperatures $T_{e}$ or differential emission measures $\operatorname{DEM}\left(T_{e}\right)=N_{e}^{2} d V / d T_{e}$ (where $N_{e}=$ electron density and $V=$ emitting volume), and from the other line ratios the abundance ratios $\mathrm{K} / \mathrm{Ar}$ and $\mathrm{Ar} / \mathrm{S}$. The measured continuum in channels 1 and 2 in addition gave us the absolute abundances, i.e. $\mathrm{K} / \mathrm{H}, \mathrm{Ar} / \mathrm{H}$, and $\mathrm{S} / \mathrm{H}$. For each time interval chosen, total count rates in each line were estimated by fitting Gaussians to the line profiles.

Figure 1 shows measured line count rates, line-to-line and line-to-continuum ratios for the flare of 2002 July 26/27. For this flare, RESIK observations lasted from 21:11 UT (July 26) to 04:06 UT (July 27), with the Ar XVIII lines measurable until 23:23 UT (July 26). Also shown are the GOES 1-8 $\AA$ light curve and values of $T_{0}$ from model (C). The observed Ar XVIII/K XVIII count rate ratios divided by the expected ratios for this flare have a mean of $1.7 \pm 0.5$ for model (A), $0.6 \pm 0.2$ for model (B), and $0.3 \pm 0.1$ for model (C) (uncertainties are standard deviations). The equivalent ratios for the Ar XVII/S XV are $0.9 \pm 0.2$ for model (A), $0.7 \pm 0.1$ for model (B), and $0.8 \pm 0.1$ for model (C). There is no significant variation over the time of the flare decay.

There are thus differences in the ratios from the different models. We can discriminate between the models to some extent by the consistency of the $\mathrm{Ar} / \mathrm{H}$ ratio, determined from the Ar XVIII/continuum ratio (channel 1) and the Ar 


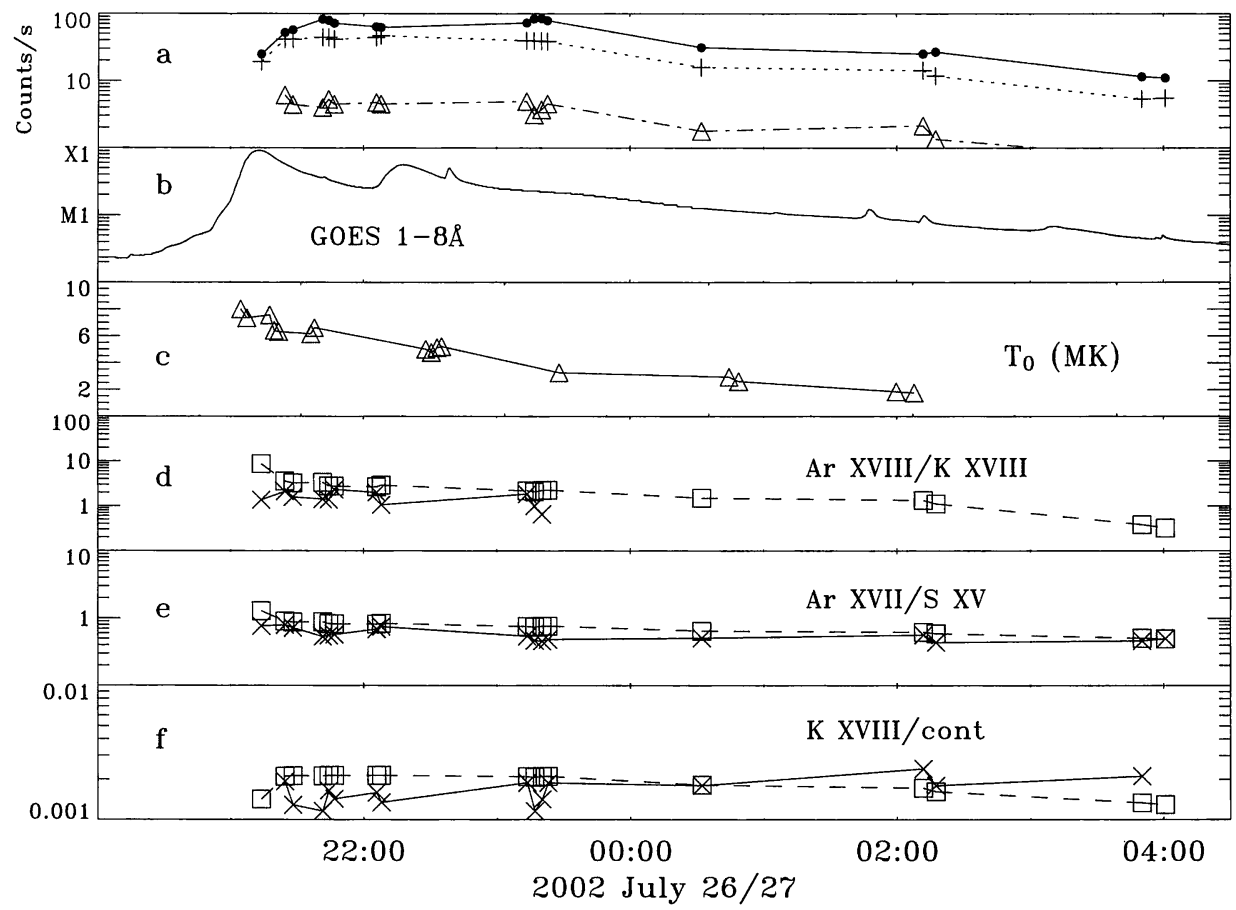

Figure 1. (a) Total RESIK count rates $\left(\mathrm{s}^{-1}\right)$ in the S XV lines (4.09, $4.30 \AA$, filled circles); Ar XVII $w$ line (3.95 $\AA$, plus signs); and K XVIII $w$ line (3.53 $\AA$, triangles) during the 2002 July $26 / 27$ flare. (b) GOES 1-8 $\AA$ X-ray light curve. (c) Values of $T_{0}$ in DEM model (C). $(d)$ Observed (crosses) and expected (squares) of the Ar XVIII/K XVIII line ratio (photon units). (e) Same as $(d)$ but for the Ar XVII/S XV line ratio. $(f)$ Observed (crosses) and expected (squares) values of the $\mathrm{K}$ XVIII/continuum ratio. The expected ratios are calculated with DEM model $(\mathrm{C})$ and coronal element abundances.

XVII ( $w$ line)/continuum ratio (channel 2). We first made corrections for the small amount of instrumental background in channel $1(3.2 \%)$ and channel 2 $(0.2 \%)$. We found that model (C) gave better consistency for the $\mathrm{Ar} / \mathrm{H}$ ratio, with the observed Ar XVIII/continuum ratios less by a factor $2.5 \pm 0.7$ than the expected ratios, and the observed Ar XVII/continuum ratios less by a factor $1.4 \pm 0.1$ than the expected ratios. For models (A) and (B) there was much less consistency between the two ratios.

\section{Conclusions}

Taking the differential emission measure model $(\mathrm{C})$ to be the most reliable of the three differential emission measure models, we obtained the following abundance ratios: $\mathrm{Ar} / \mathrm{K}=4.3 \pm 1.5 ; \mathrm{Ar} / \mathrm{S}=0.15 \pm 0.03 ; \mathrm{Ar} / \mathrm{H}=(1.5 \pm 0.4) \times 10^{-6}$ (from 
Ar XVIII/continuum); $\mathrm{Ar} / \mathrm{H}=(2.8 \pm 0.2) \times 10^{-6}$ (from Ar XVII/continuum); $\mathrm{K} / \mathrm{H}=(3.7 \pm 1.0) \times 10^{-7} ;$ and $\mathrm{S} / \mathrm{H}=(2.2 \pm 0.4) \times 10^{-5}$.

Very similar (at most $30 \%$ different) values were determined for these ratios from measurements during the flares of April 14/15, July 11, and July 22/23, except for the $\mathrm{S} / \mathrm{H}$ and $\mathrm{Ar} / \mathrm{S}$ ratios during the April 14/15 and July 11 flares which indicate a lower (by a factor 2) $\mathrm{S}$ abundance. For both these flares, the $\mathrm{S}$ XV 4.299 $\AA$ line was outside the range of channel 2 owing to the flare position on the Sun relative to the crystal dispersion direction, so only the weaker $4.088 \AA$ was available. We therefore believe the measurements of the July $22 / 23$ and July $26 / 27$ flares to be more reliable. There is in addition supporting evidence from a channel 1 spectrum summed over a period near the maximum of the July $26 / 27$ flare when the S XVI Ly $\delta$ line $(3.693 \AA)$ was visible, giving an Ar/S ratio consistent with the $\mathrm{S}$ abundance from this flare.

Our model $(\mathrm{C}) \mathrm{K} / \mathrm{H}$ abundance ratio $\left((3.7 \pm 1.0) \times 10^{-7}\right)$ is consistent with values obtained by Doschek et al. (1985) $\left(4.2 \times 10^{-7}\right)$ and Landi et al. (2002) $\left(4.7 \pm 10^{-7}\right)$, but is a factor 3 more than the photospheric value (Takeda et al. 1996). The $\mathrm{S} / \mathrm{H}$ abundance ratio $\left((2.2 \pm 0.4) \times 10^{-5}\right)$ agrees with the coronal value of Feldman \& Laming (2000) and the photospheric value of Grevesse \& Sauval (1998). The $\mathrm{Ar} / \mathrm{H}$ abundance ratio $\left((2.8 \pm 0.2) \times 10^{-6}\right)$ is consistent with the energetic particles value (Reames 1998) and slightly lower than the value obtained from an impulsive flare with photospheric abundances $((3.9 \pm 1.1) \times$ $10^{-6}$ : Feldman \& Laming 2000).

In summary, our results fit a pattern in which low-FIP elements are enriched by a factor 3 and with high-FIP elements (including S) having abundances equal to photospheric. Potassium has the lowest FIP of any abundant element in the Sun, yet the enrichment factor does not appear to be larger than 3 , ruling out the possibility that coronal/photospheric enhancements increase with decreasing FIP, as might be deduced from the plot given (as Fig. 13) by Feldman \& Laming (2000).

\section{References}

Dere, K. P., Landi, E., Mason, H. E., Monsignori Fossi, B. C., \& Young, P. R. 1997, A\&AS, 125, 149

Doschek, G. A., Feldman, U., \& Seely, J. F. 1985, MNRAS, 217, 317

Feldman, U., \& Laming, J. M. 2000, Phys. Scr., 61, 222

Grevesse, N., \& Sauval, A. J. 1998, Space Sci. Rev., 85, 161

Landi, E., Feldman, U., \& Dere, K. P. 2002, ApJS, 139, 281

Landi, E., Feldman, U., Innes, D. E., \& Curdt, W. 2003, ApJ, 582, 506

Mazzotta, P., Mazzitelli, G., Colafranscesco, S., \& Vittorio, N. 1998, A\&AS, 133,403

Reames, D. V. 1998, Space Sci. Rev., 85, 327

Takeda, Y., Kato, K.-I., Watanabe, Y., \& Sadakane, K. 1996, PASJ, 48, 511 